\title{
Relation between serum zinc levels and recurrent urinary tract infections in female patients: A case-control study
}

\author{
Behzad Mohsenpour ${ }^{1,2}$, Amjad Ahmadi*3, Anvar Mohammadi Baneh4, Katayoon Hajibagheri, ${ }^{1,2}$, \\ Ebrahim Ghaderi ${ }^{1,5}$, Shahla Afrasiabian ${ }^{1,2}$, Samaneh Azizi ${ }^{6}$

\begin{abstract}
Background: Zinc deficiency can increase the risk of infectious diseases. Given that recurrent urinary tract infection is a common complication, this study examines the association between serum zinc levels and recurrent urinary tract infections.

Methods: In this case-control study, serum zinc levels for 48 patients with recurrent urinary tract infections were compared with the serum zinc levels of the same number of people in the control group who were matched in terms of location and age. Using SPSS ver. 18, univariate analysis was performed through t-test, correlation coefficient; and multivariate analysis was carried out through multiple regression tests. Significant level was considered as less than 0.05 .

Results: There was a weak correlation between age and serum zinc level $(\mathrm{r}=-0.205, \mathrm{p}=0.045)$. Mean serum zinc level of the test group and the control group were $96.83( \pm 11.25)$ and $76.72( \pm 17.06)$ microgram/deciliter $(\mathrm{p}=0.001)$, respectively. Level of zinc reduced with aging; in addition, the group with recurrent UTIs had lower zinc levels than the control group $\left(p=0.010, R^{2}=0.377\right.$ ).

Conclusion: According to the results of this study, serum zinc levels of people with recurrent urinary tract infections were lower than that in the control group. It seems that zinc levels are a risk factor for recurrent urinary tract infections.
\end{abstract}

Keywords: Urinary tract infection, Micronutrients, Zinc, Recurrent infections, Nutrition

Conflicts of Interest: None declared

Funding: Kurdistan University of Medical Sciences and Research Deputy of Kurdistan University of Medical Sciences

*This work has been published under CC BY-NC-SA 1.0 license.

Copyright $\odot$ Iran University of Medical Sciences

Cite this article as: Mohsenpour B, Ahmadi A, Mohammadi Baneh A, Hajibagheri K, Ghaderi E, Afrasiabian Sh, Azizi S. Relation between serum zinc levels and recurrent urinary tract infections in female patients: A case-control study. Med J Islam Repub Iran. 2019 (22 Apr);33:33. https://doi.org/10.47176/mjiri.33.33

\section{Introduction}

Urinary tract infections (UTIs) are more common in women and become more prevalent with age (1-3). Prevalence of UTIs is $10 \%$ among people aged 4-7 years old while it is about $52 \%$ in people aged $18-26$ years old $(4,5)$. Recurrence is one of the complications of the disease, and its prevalence is different. In a follow-up study, the recurrent disease was observed once in $27 \%$ of patients and twice in $2.7 \%$ of UTI patients. Recurrent UTIs are defined as incidences of UTIs at least twice in six months or three or more times in one year (6).

Corresponding author: Amjad Ahmadi, microbiology90@gmail.com

Zoonoses Research Center, Kurdistan University of Medical Sciences, Sanandaj, Iran

2. Department of Infectious Diseases, Faculty of Medicine, Kurdistan University of Medical Sciences, Sanandaj, Iran

3. Department of Microbiology, Faculty of Medicine, Kurdistan University of Medical Sciences, Sanandaj, Iran

4. Department of Internal Medicine, Faculty of Medicine, Kurdistan University of Medical Sciences, Sanandaj, Iran

5. Social Determinants of Health Research Center, Research Institute for Health Development, Kurdistan University of Medical Sciences, Sanandaj, Iran

6. Students Research Committee, Kurdistan University of Medical Sciences, Sanandaj, Iran
Zinc is a micronutrient, and its deficiency can increase the risk of infectious diseases (7). Zinc is involved in the regulation of the host immune system, and its moderate deficiency leads to the dysfunction of the immune system. Zinc is necessary for the development and activity of $\mathrm{T}$ lymphocytes, and its deficiency leads to decreased levels of cellular immunity (8). Host cells can change the cytoplasmic and lysosomal level of zinc to make a response to the bacteria; in addition, the accumulation of zinc in phagolysosome and macrophages helps to control patho-

$\uparrow$ What is "already known" in this topic:

According to the results of this study, It seems that serum zinc level is a risk factor for recurrent urinary tract infections.

$\rightarrow$ What this article adds:

There is a relationship between low serum zinc level and urinary tract infection. Therefore, a clinical trial is required for people with UTIs. 
gens. The bacterial poisoning caused by zinc can be a defense mechanism used by macrophages to clear the infection (9). Zinc deficiency is associated with bad outcomes in response to bacterial infections and sepsis (10). So, it seems that zinc levels can be a risk factor for recurrent UTIs. This study compared serum zinc levels between women with and without recurrent UTIs.

\section{Methods}

This case-control study was conducted after aprooval by the Ethics Committee of Kurdistan University of Medical Sciences (MUK.REC.1392.158). Considering a 1\% error rate of 0.05 , mean zinc level of $105.9( \pm 23.7)$ in healthy subjects and $95.4( \pm 21.8)$ in patients, and second type error of $10 \%$, sample size was calculated as 48 in each group (10). To enroll the subjects into the two groups, they were briefed about the objectives of the study and informed consent was obtained. The study participants included women with recurrent UTIs who referred to Tohid Hospital or the Clinic of Infectious Diseases. Initial screening was performed by an infectious diseases specialist who examined patients to detect signs and symptoms of UTIs.

UTIs are defined as: presence of dysuria, or/and frequency, and suprapubic pain or/and tenderness, or/and costovertebral tenderness, or/and fever, or/and chills with WBC equal or more than $10 / \mathrm{hpf}$ in centrifuged urine smear and presence of organism compatible with UTI more than 100000 in urine culture. After initial screening, the inclusion criteria were defined as recurrent UTIs or UTI at least twice in the last six months, or UTI at least three times over the past year, and being older than 12 years. It is worth noting that only incidences were enrolled into the study (i.e. those who had not been treated for recent infection). Considering the exclusion criteria, we did not enroll those who consumed zinc in the past year, immunocompromised patients, dialysis patients, patients receiving corticosteroid therapy, patients with cancer, and patients with malabsorption.

The control group consisted of those referred to clinics to undergo routine tests or to accompany patients and consented to participate in this study. They underwent primary screening, which was performed by an infectious disease specialist; in the absence of UTI symptoms or a history of past UTIs, they were selected as members of the control group. The control group matched with the cases in terms of age and place of residence (urban/rural) by group matching. To ensure the absence of UTIs, participants in the control group underwent urinalysis and a urine culture test. The exclusion criteria used for the control group were similar to those used for test group.

Blood samples $(5 \mathrm{cc})$ were taken from the subjects who met the inclusion criteria; to measure the serum level, the collected blood samples were sent to the laboratory. The subjects in the control group underwent the same procedure and the tests were performed by one laboratory. To measure the serum zinc levels, a zinc calorimetry test was performed using the BT 3000 auto analyzer device (made in Italy) and the Bio rex kit (made in the UK).

\section{Statistical analysis}

After entering the collected data into SPSS ver. 18, charts and tables were used to describe the variables. A Chi-square test and Fisher test were used to compare qualitative variables between the two groups; moreover, the ttest and Pearson correlation coefficient were used to compare quantitative values. Then, multiple linear regression was used to determine factors affecting serum zinc levels. The significance level was set at 0.05 .

\section{Results}

The mean age of the participants in the test group and the control groups was $53.37( \pm 19.2)$ and $52.70( \pm 19.33)$ years, respectively $(\mathrm{p}=0.865)$. The mean serum zinc levels of the participants from urban and rural areas were 86.78 $( \pm 17.32)$ and $86.75( \pm 20.6)$ microgram/deciliter, respectively $(\mathrm{p}=0.995)$. There was a weak correlation between age and serum zinc level $(\mathrm{r}=-0.205, \mathrm{p}=0.045)$. Mean serum zinc level of the test group and the control group was $96.83( \pm 11.25)$ and $76.72( \pm 17.06)$ microgram/deciliter $(\mathrm{p}=0.001)$, respectively.

The results of multiple linear regression (Table 1) shows that the level of zinc reduced with age; in addition, the group with recurrent UTIs had lower zinc levels than the control group $\left(\mathrm{p}=0.010, \mathrm{R}^{2}=0.377\right)$.

Decreased serum zinc levels was related to increased age and recurrent infections after adjusting for other variables.

\section{Discussion}

According to the results of this study, people with recurrent UTIs had lower serum zinc levels than the control group; this difference was also observed after adjusting for age and location. Javadinia et al. (11) showed that serum zinc levels were significantly lower in children with UTIs than in the control group. In our study, we observed the same result. This confirms that serum zinc levels in patients with UTIs are lower than healthy individuals. It should be noted that our study was conducted on patients with recurrent UTIs, which was different from Javadinia et al. (11). The results of our study showed that serum zinc levels decreased with age and it was observed in both groups, which indicates a reverse relationship between age and serum zinc level. Given the fact that with aging there

\begin{tabular}{|c|c|c|c|c|c|c|}
\hline \multirow[t]{2}{*}{ Model } & \multicolumn{2}{|c|}{ Non-standard coefficients } & \multirow{2}{*}{$\begin{array}{c}\text { Standard coefficients } \\
\text { Beta Coefficient }\end{array}$} & \multirow[t]{2}{*}{$\mathrm{p}$} & \multicolumn{2}{|c|}{$95 \% \mathrm{CI}$ for $\mathrm{B}$} \\
\hline & Beta Coefficient & Std. Error & & & Lower bound & Upper bound \\
\hline Constant coefficient & 107.82 & 6.75 & & $<0.001$ & 94.40 & 121.23 \\
\hline Age (years) & -0.198 & 0.076 & -0.215 & 0.010 & -0.34 & -0.04 \\
\hline Location (urban / rural) & -0.384 & 4.35 & -0.007 & 0.930 & -9.02 & 8.25 \\
\hline Group (recurrent infection / control) & -20.23 & 2.87 & -0.579 & 0.001 & -25.95 & -14.52 \\
\hline
\end{tabular}


is an increase in the incidence of UTIs, it can be hypothesized that, in addition to various causes for the increased incidence of UTIs in old age, zinc deficiency can also be regarded as one of the reasons.

Hancock et al. (12) indicated that the antimicrobial and anti-biofilm effects of zinc on urinary tract pathogens, E.coli, and Klebsiella was investigated; it was observed that the divalent zinc was able to inhibit the mechanisms of biofilm formation by the studied organisms in order to apply its antimicrobial mechanism. Therefore, it might be concluded that frequent recurrences may be due to the formation of biofilm in the urinary tract or stones in the urinary system.

Combination of some antibiotics with zinc results in a synergistic effect against organisms and might be effective in decreasing recurrent infections. Zinc is a cofactor of more than 200 enzymes that are necessary for the metabolic activity of cells. Additionally, zinc has an important role in immune system physiology and is important for the development and activity of $\mathrm{T}$ lymphocyte. Decreased serum zinc levels cause decreased immune system activity. Zinc has an important role to play in the production of gamma interferon, interleukin-2, and interferon alpha. Zinc is necessary for radical detoxification and antioxidant defenses. In patients with UTIs, oxidative stress is increased and antioxidant levels is decreased. Markers of oxidative stress such as malondialdehyde in UTIs is increased; whereas, serum cations such as $\mathrm{Cu}, \mathrm{Ca}$, and $\mathrm{Zn}$ are decreased (13).

The above-mentioned conclusions are consistent with the results of our study. The administration of antibiotics together with an appropriate level of serum zinc could result in a synergistic ability to eradicate organisms in the urinary system.

This study had some limitations. We could not follow patients with low levels of serum zinc. Follow up of these patients can clearly define the possible relation of zinc deficiency and recurrent UTI.

\section{Conclusion}

It can be suggested that recurrent UTIs are associated with low serum zinc levels. Therefore, it is recommended to conduct a clinical trial to study the effects of zinc supplementation on patients with recurrent UTIs and to determine their effectiveness in the prevention or treatment of recurrent UTIs.

\section{Acknowledgments}

This paper was extracted from a thesis belongs to Samaneh Azizi and would like to thank Kurdistan University of Medical Sciences and Research Deputy of Kurdistan University of Medical Sciences for financial support.

\section{Conflict of Interests}

The authors declare that they have no competing interests.
2. Timur-Taşhan S, Beji NK, Aslan E, Yalçin Ö. Determining lower urinary tract symptoms and associated risk factors in young women. International journal of gynaecology and obstetrics. Int $\mathrm{J}$ Gynaecol Obstet. 2012;118:27-30.

3. Foxman B. Urinary tract infection syndromes: occurrence, recurrence, bacteriology, risk factors, and disease burden. Infect Dis North Am. 2014;28:1-13.

4. Kyrklund K, Taskinen S, Rintala RJ, Pakarinen MP. Lower urinary tract symptoms from childhood to adulthood: a population based study of 594 Finnish individuals 4 to 26 years old. J Urol. 2012:188:588-93.

5. Kodner CM, Thomas Gupton EK. Recurrent urinary tract infections in women: diagnosis and management. Am Fam Physician. 2010;82:638-43.

6. Syukri M, Imran I, Harapan H, Sja'bani M, Astuti I, Soesatyo MH. Comparison of serum $\mathrm{C} 3$ complement levels between young women with recurrent urinary tract infection and healthy women. Alexandria J Med. 2015;51:35-9.

7. Sampaio DL, De Mattos ÂP, Ribeiro TCM, Leite MEDQ, Cole CR, Costa-Ribeiro Jr H. Zinc and other micronutrients supplementation through the use of sprinkles: impact on the occurrence of diarrhea and respiratory infections in institutionalized children. J Pediatr. 2013;89:286-93

8. Yousefi P, Moghaddasi Z, Tabaei A. Therapeutic effects of zinc supplementation in children with urinary tract infection. Koomesh. 2011;15:204-8.

9. Botella H, Peyron P, Levillain F, et al. Mycobacterial p(1)-type ATPases mediate resistance to zinc poisoning in human macrophages. Cell Host Microbe 2011;10:248-59.

10. Liu MJ, Bao S, Gálvez-Peralta M, et al. ZIP8 regulates host defense through zinc-mediated inhibition of NF-kappaB. Cell Rep. 2013;3:386-400

11. Javadi Nia S, Noorbakhsh S, Izadi A, Tabatabaei A, Shokrollahi MR. Comparison of vitamin A, D \& zinc serum levels between children with urinary tract infection and control group in two University Hospital. Tehran Uni Med J. 2013;71:244-9.

12. Hancock V, Dahl M, Klemm P. Abolition of biofilm formation in urinary tract Escherichia coli and Klebsiella isolates by metal interference through competition for fur. Appl Environ Microb. 2010;76:3836-41.

13. Elkhatib W, Noreddin A. In Vitro Antibiofilm Efficacies of Different Antibiotic Combinations with Zinc Sulfate against Pseudomonas aeruginosa Recovered from Hospitalized Patients with Urinary Tract Infection. Antibiotics. 2014;3:64-84.

\section{References}

1. Foxman B. Epidemiology of urinary tract infections: incidence, morbidity, and economic costs. Am J Med. 2002;113:5S-13S. 